\title{
Isoform Switching of Type IV Collagen Is Developmentally Arrested In X-Linked Alport Syndrome Leading to Increased Susceptibility of Renal Basement Membranes to Endoproteolysis
}

\author{
Raghuram Kalluri, ${ }^{\star}$ Charles F. Shield III,‡ Parvin Todd, $§$ Billy G. Hudson, ${ }^{\S}$ and Eric G. Neilson* \\ *Penn Center for Molecular Studies of Kidney Diseases, Renal Electrolyte and Hypertension Division, University of Pennsylvania \\ Medical School, Philadelphia, Pennsylvania 19104-6144; ${ }^{\ddagger}$ St. Francis Regional Medical Center, Wichita, Kansas 67214; and ${ }^{\S}$ Department \\ of Biochemistry and Molecular Biology, University of Kansas Medical Center, Kansas City, Kansas 66160
}

\begin{abstract}
Normal glomerular capillaries filter plasma through a basement membrane (GBM) rich in $\alpha 3$ (IV), $\alpha 4$ (IV), and $\alpha 5$ (IV) chains of type IV collagen. We now show that these latter isoforms are absent biochemically from the glomeruli in patients with X-linked Alport syndrome (XAS). Their GBM instead retain a fetal distribution of $\alpha 1$ (IV) and $\alpha 2$ (IV) isoforms because they fail to developmentally switch their $\alpha$-chain use. The anomalous persistence of these fetal isoforms of type IV collagen in the GBM in XAS also confers an unexpected increase in susceptibility to proteolytic attack by collagenases and cathepsins. The incorporation of cysteine-rich $\alpha 3$ (IV), $\alpha 4($ IV), and $\alpha 5$ (IV) chains into specialized basement membranes like the GBM may have normally evolved to protectively enhance their resistance to proteolytic degradation at the site of glomerular filtration. The relative absence of these potentially protective collagen IV isoforms in GBM from XAS may explain the progressive basement membrane splitting and increased damage as these kidneys deteriorate. (J. Clin. Invest. 1997. 99:24702478.) Key words: Alport • proteases - type IV collagen • basement membrane $\bullet$ kidney
\end{abstract}

\section{Introduction}

The average human kidney filters over 70 liters of plasma water and solute each day through $10^{6}$ glomerular tufts (1). This enormous work load is performed by specialized capillaries with fenestrated endothelium on the blood side separated by a glomerular basement membrane $(\mathrm{GBM})^{1}$ from external epithelial podocytes and slit-pores that allow passage of ultrafiltrate into the urinary space $(2,3)$. Traditional basement

Address correspondence to Eric G. Neilson, M.D., C. Mahlon Kline Professor of Medicine and Pediatrics, Penn Center for Molecular Studies of Kidney Diseases, 700 Clinical Research Building, University of Pennsylvania, 415 Curie Boulevard, Philadelphia, PA 191046144. Phone: 215-662-7633; FAX: 215-898-0189; E-mail: neilson@ mail.med.upenn.edu

Received for publication 14 November 1996 and accepted in revised form 5 March 1997.

1. Abbreviations used in this paper: GBM, glomerular basement membrane; $\mathrm{NC1}$, COOH-terminal noncollagenous globular domain; RBM, renal basement membrane; XAS, X-linked Alport Syndrome.

J. Clin. Invest.

(C) The American Society for Clinical Investigation, Inc. 0021-9738/97/05/2470/09 \$2.00

Volume 99, Number 10, May 1997, 2470-2478 membranes are composed of type IV collagens, laminins, proteoglycans, and entactin (4). GBM is a highly specialized basement membrane with $\alpha 3$ (IV), $\alpha 4$ (IV), and $\alpha 5$ (IV) collagens represented much more abundantly than $\alpha 1$ (IV) and $\alpha 2$ (IV) chains $(5,6)$. Basement membranes in placenta and liver exhibit a limited diversity in collagen composition and are principally formed of $\alpha 1$ (IV) and $\alpha 2$ (IV) collagens (5). The specialization of basement membrane, when it occurs, likely serves a unique organ function, although the environmental drivers of this developmental switch are not known.

Type IV collagen is a family of complex polypeptides (5). The $\alpha 1$ (IV) and $\alpha 2(\mathrm{IV})$ chains are products of distinct genes located pairwise in a head-to-head fashion on chromosome 13 (5). $\alpha 3(\mathrm{IV})$ and $\alpha 4(\mathrm{IV})$ chains are present in the same orientation on chromosome 2 , and the $\alpha 5$ (IV) and $\alpha 6$ (IV) chains are located on the X-chromosome (5). Type IV collagen protomers consist of three $\alpha$-chains that come together by covalent and noncovalent interactions to form a triple helical structure (5). The protomer is divided into three different domains, an $\mathrm{NH}_{2}$-terminal $7 \mathrm{~S}$ domain, a middle triple helical domain, and a $\mathrm{COOH}$-terminal noncollagenous globular domain (NC1) (5). Each protomer is bonded with another protomer by its $\mathrm{NC1}$ domain to form interlocking hexamers (5). With six different $\alpha$-chains known at present, several different combinations of triple helical protomers and hexamers are possible (7).

One interesting natural mutation of basement membrane collagen is found in Alport syndrome (8). About $80 \%$ of the families with this hereditary nephritis show an X-linked inheritance (XAS) of mutations in the COL $4 \alpha 5$ gene encoding the $\alpha 5$ (IV) chain, while some of the remainder inherit autosomal recessive mutations in $\alpha 3$ (IV) or $\alpha 4$ (IV) chains $(5,8)$. Most males with XAS have near normal kidneys at birth $(9,10)$. However, over time their kidneys develop splitting of the GBM and interstitial fibrosis leading to renal failure (11). Recent studies using chain-specific antibodies as indirect structural probes suggest that not only is the mutated $\alpha 5$ (IV) chain not expressed in XAS, but also that $\alpha 3$ (IV) and $\alpha 4$ (IV) collagens may be absent $(12,13)$. The degree to which these other chains are absent may depend on how a mutation effects either transcriptional (12) or posttranslational events (14).

Finally, GBM, like other basement membranes, is continuously remodeled in vivo and vulnerable to the actions of local proteases (15-24). Since glomerular capillaries filter plasma water, the concentration of serum endopeptidases, like other proteins, increases transiently on passage through the glomerular microenvironment adjacent to the GBM. Although endopeptidases seem to have some selectivity for different basement membrane moieties, it is not yet known whether isoforms of the same constituent are equally resistant to the same enzyme. We hypothesize that the collagens in normal kidney basement membrane are more resistant to proteases 
than membranes from XAS, and we have used quantitative methodology to compare their degradation.

\section{Methods}

$X A S$ and embryonic kidneys. Nonfunctioning XAS kidneys were obtained from four different male Alport patients in whom it was deemed appropriate to remove the native organ at surgery, as well as one kidney each from a diabetic and a patient with IgA nephropathy. Timed embryonic kidney tissue was also obtained from the Laboratory for Human Embryology at the University of Washington (Seattle, WA).

Antibodies. Rabbit antibodies were prepared and characterized to all six $\alpha$-chains of type IV collagen as recombinant molecules (7, 25, 26). Alport alloantibodies and Goodpasture autoantibodies were also described previously $(7,27)$.

Preparation of basement membrane and type IV collagen domains. Kidney cortexes were shaved into protease inhibitors, homogenized, and suspended in $2 \%$ deoxycholate and stirred for $4 \mathrm{~h}$ at room temperature (28). The suspension was centrifuged, washed three times with ice cold distilled water, each time homogenizing with a polytron homogenizer (28). To solubilize the noncollagenous domain of collagen IV, renal basement membrane preparations were digested with bacterial collagenase at $37^{\circ} \mathrm{C}$ for $24 \mathrm{~h}$ in the following digestion buffer: $0.05 \mathrm{M}$ Hepes, pH 7.5, $0.01 \mathrm{M} \mathrm{CaCl}_{2}, 4 \mathrm{mM} \mathrm{N}$-ethylmaleimide, $5 \mathrm{mM}$ benzamidine $\mathrm{HCl}, 1 \mathrm{mM}$ phenylmethanesulfonyl fluoride, and $25 \mathrm{mM}$ e-aminohexanoic acid $(7,29)$.

Anion-exchange and reverse-phase chromatography. After treatment with collagenase, purification of the noncollagenous domain (hexamer) was carried out under associative conditions using anionexchange chromatography on a DEAE-52 column (Millipore Corp. Milford, MA) in $50 \mathrm{mM}$ Tris- $\mathrm{HCl}$ buffer at $\mathrm{pH} 7.5$ (7). The main unbound peak, which contained the noncollagenous domains, was concentrated by ultrafiltration (YM-10 filter, Amicon, Inc., Beverly, MA). The pooled proteins were applied to a C-18 reverse-phase column $(4.6 \mathrm{~mm} \times 25 \mathrm{~cm})$. The column was developed in a gradient starting from $0.1 \%$ trifluroacetic acid and $31 \%$ acetonitrile to $0.1 \%$ trifluroacetic acid and $39 \%$ acetonitrile. The gradient was created over a period of $48 \mathrm{~min}$ at $1.2 \mathrm{ml} / \mathrm{min}$. Alternatively, a 30-min gradient was also run at $2.0 \mathrm{ml} / \mathrm{min}$.

SDS-PAGE electrophoresis. SDS-PAGE in one and two dimensions was carried out with $10-20 \%$ or $4-22 \%$ linear gradient gels and discontinuous buffers (7). Electrophoresis in the first dimension of the two-dimensional electrophoresis was performed as nonequilibrium $\mathrm{pH}$ gel electrophoresis (NEPHGE) (30), and in the second dimension $10-20 \%$ gradient gels were used in SDS-PAGE. NEPHGE was carried out at $8^{\circ} \mathrm{C}$ for $3,000 \mathrm{~V} / \mathrm{h}$. Molecular weight markers for the second dimension were used to verify the migration of proteins through the gel and identify the protein spots originating from the NC1 hexamer and $\alpha 3$ (IV), $\alpha 4($ IV), $\alpha 5$ (IV), and $\alpha 6$ (IV) dimers.

Western blotting. Western blotting was performed for one- and two-dimensional blotting $(7,31,32)$, the separated proteins were transferred to nitrocellulose or immobilon paper and blocked with $2 \% \mathrm{BSA}$ for $30 \mathrm{~min}$ on a shaker at room temperature. After blocking the remaining binding sites, the blot paper was washed thoroughly with washing buffer and incubated with a primary antibody at an appropriate dilution in PBS containing 1\% BSA. Incubation was carried out at room temperature overnight on a shaker. Subsequently, the blot was washed thoroughly with washing buffer and incubated with a secondary antibody conjugated to horseradish peroxidase for $3 \mathrm{~h}$ at room temperature on a shaker. The blot was again washed thoroughly and substrate (diaminobenzidine in $0.05 \mathrm{M}$ phosphate buffer containing $0.01 \%$ cobalt chloride and nickel ammonium) was added and incubated for $10 \mathrm{~min}$ at room temperature. Upon completion of this step, the substrate was poured out and substrate buffer containing hydrogen peroxide was added. After development of bands, the reaction was stopped with distilled water and the blot was dried on paper towels.
Direct ELISA assay. The ELISA plates were coated in triplicate with $200 \mathrm{ng}$ antigen in $200 \mu \mathrm{l}$ coating buffer (33). The plates were incubated for $2 \mathrm{~h}$ at $37^{\circ} \mathrm{C}$ or overnight at room temperature. Upon coating, the plates were washed three times at intervals of $5 \mathrm{~min}$, with washing buffer, blocked with 1 or $2 \% \mathrm{BSA}$ for $30 \mathrm{~min}$ at $37^{\circ} \mathrm{C}$, washed again with the washing buffer, and then incubated with primary antibody in appropriate dilution in PBS containing 1\% BSA. The plates were incubated for $1 \mathrm{~h}$ at room temperature. After primary antibody incubation, the plates were again washed and subsequently incubated with a secondary antibody conjugated to alkaline phosphatase diluted 1:5,000 in incubation buffer. The plates were incubated for $1 \mathrm{~h}$ at room temperature. Subsequently, the plates were washed thoroughly and substrate, disodium $p$-nitrophenyl phosphate $(5 \mathrm{mg} / \mathrm{ml})$ was added. After color development, the absorbance at 410 or $405 \mathrm{~nm}$ was measured using a microplate reader.

Immunofluorescence and immunohistochemistry. Formalin-fixed tissue was paraffin embedded and $4-\mu \mathrm{m}$ sections were obtained. Sections were stained with periodic acid Schiff and/or hematoxylin and eosin for pathological analysis. Immunofluorescence and immunoperoxidase stainings were performed as previously described $(34,35)$.

Kidney basement membrane digestion and hydroxyproline release assay. Renal basement membrane (RBM) and NC1 hexamer digestions with bacterial collagenase, Pseudomonas elastase, cathepsin B, and cathepsin $\mathrm{G}$ were performed with gentle shaking at either $25^{\circ} \mathrm{C}$ (elastin and cathepsin B) or $37^{\circ} \mathrm{C}$ (collagenase and cathepsin $\left.\mathrm{G}\right)(7$, $18,19,36,37)$. For bacterial collagenase, $18.5 \mathrm{U}$ enzyme was used to digest $1 \mathrm{mg}$ of detergent-extracted RBM over a 2-h interval. $2 \mathrm{U}$ cathepsin $\mathrm{B}, 0.10 \mathrm{U}$ cathepsin $\mathrm{G}$, and $20 \mathrm{U}$ P. elastase were used for similar RBM digestions. Hydroxyproline assays were performed as described by Huszar et al. (36). For hydroxyproline release assays using conditioned medium from glomerular cell cultures, we used the methods described by Knowlden et al. (24), Martin et al. (37), and Lovett et al. (38) to collect conditioned medium from confluent rat glomerular epithelial $(34,35)$, glomerular endothelial (14), and mesangial cells (33). Two million cells of each type were grown to confluence in 5-ml vol. Equal volumes of supernatants from all three glomerular cell types were pooled, supplemented with $2 \mathrm{mM}$ of $\mathrm{CaCl}_{2}$, and then used as media for incubating detergent-extracted RBM from normal, Alport, diabetic, and IgA nephropathy kidneys. In three experiments, $1 \mathrm{mg}$ of RBM from each test kidney was placed in $15 \mathrm{ml}$ of pooled, conditioned medium for $12 \mathrm{~h}$ with gentle shaking at $37^{\circ} \mathrm{C} .20 \mathrm{mM}$ EDTA was added at the end of incubation to chelate calcium and stop the reaction. The supernatants and pellets were collected for measurement of hydroxyproline release. Each set of experiments was repeated three times.

Statistics. In some experiments, statistics were performed using Student's $t$ test.

\section{Results}

Human kidney begins developing after $40 \mathrm{~d}$ of fetal life. Fetal kidneys from 50-, 75-, or 150-d postcoitus were stained with antibodies specific for all six chains of type IV collagen as well as Goodpasture (GP-1/GP-2) and transplant alloantisera (ALP-1/ ALP-2) that recognizes novel epitopes on the $\alpha 3(\mathrm{IV})$ chain. Glomeruli were examined at three levels from the cortical surface to deep nephrons; the newly forming cortical nephrons including the comma and S-shaped bodies, the early glomerular capillary loop nephrons, and the mature glomerulus. As seen in Table I, $\alpha 1$ and $\alpha 2$ chains are present in the early stages of nephron development but are replaced in glomeruli by $\alpha 3$ (IV), $\alpha 4(\mathrm{IV})$, and $\alpha 5$ (IV) collagens in mature capillary loops; $\alpha 6$ (IV) appears only in Bowman's capsule. Fig. 1 shows fetal human kidney (75 d) stained with antibodies directed against $\alpha 1(\mathrm{IV})$, $\alpha 3$ (IV) and $\alpha 5$ (IV) collagens. The antibody to $\alpha 1$ (IV) chain reacts with basement membrane in early cleft/S-shaped bodies, 
Table I. Immunohistochemical Staining for Type IV Collagen in Fetal Human Kidney

\begin{tabular}{|c|c|c|c|c|c|}
\hline \multirow[b]{2}{*}{ Antibody } & \multirow[b]{2}{*}{$\begin{array}{l}\text { Newly formed } \\
\text { nephrons }\end{array}$} & \multirow[b]{2}{*}{$\begin{array}{l}\text { Maturing } \\
\text { nephrons }\end{array}$} & \multicolumn{3}{|c|}{ Mature glomeruli } \\
\hline & & & GBM & MM & $\mathrm{BCBM}$ \\
\hline$\alpha 1$ & + & + & - & + & + \\
\hline$\alpha 2$ & + & + & - & + & + \\
\hline$\alpha 3$ & - & - & + & $-/+$ & $+1-$ \\
\hline$\alpha 4$ & - & - & + & - & - \\
\hline$\alpha 5$ & - & $-1+$ & + & - & $-1+$ \\
\hline$\alpha 6$ & - & - & - & - & + \\
\hline ALP-1 & - & - & + & - & $-1+$ \\
\hline ALP-2 & - & - & + & - & $-1+$ \\
\hline GP-1 & - & - & + & - & - \\
\hline GP-2 & - & - & + & - & - \\
\hline $\mathrm{rC}$ & - & - & - & - & - \\
\hline $\mathrm{hC}$ & - & - & - & - & - \\
\hline
\end{tabular}

Alp, Alport alloantibodies; GP, Goodpasture autoantibodies; rC, rabbit control sera; hC, human control sera; GBM, glomerular basement membrane; BCBM, bowman's capsule basement membrane, MM, mesangial matrix. + , positive; - , negative, and $-/+$, weakly positive. Newly formed nephrons include S- and comma-shaped bodies.

but largely disappears in the mature glomeruli (Fig. 1, $A-C$ ). The $\alpha 3$ (IV) and $\alpha 5$ (IV) chains are not detected in early nephron or glomerular forms, but are expressed in mature GBM (Fig. $1, D-F$ and $G-I$ ).

Immunofluorescence evaluation was performed on the normal adult and XAS kidney sections using $\alpha$-chain-specific antibodies for all six chains of type IV collagen. These studies show $\alpha 1$ (IV) and $\alpha 2$ (IV) chain staining in the mesangial matrix, tubular basement membrane, Bowman's capsule basement membrane, and GBM of normal kidney (Table II). In normal adult human kidney, $\alpha 1$ (IV) and $\alpha 2$ (IV) chains are vastly diminished in the GBM. By contrast, $\alpha 1$ (IV) and $\alpha 2$ (IV) chains are abundant in the GBM of XAS kidneys. This observation is consistent with several published reports $(10,13,39)$. Antibody staining of $\alpha 3$ (IV), $\alpha 4(\mathrm{IV})$, and $\alpha 5$ (IV) chains is detected predominantly in the GBM, with occasional tubular basement membrane and Bowman's capsule staining in the normal kidney. In the XAS kidneys, these type IV chains are not detected with antibodies to recombinant protein or with Goodpasture or transplant alloantisera (Table II).

We next performed the first successful isolation of intact type IV collagen isoforms from five different human Alport kidneys, including two kidneys from the same patient, and two control kidneys. Basement membranes from each kidney were collagenase digested and separated on an anion exchange column to partially purify the noncollagenous domain of type IV collagen (7). This partially purified fraction mainly contains $\mathrm{NC1}$ and $7 \mathrm{~S}$ domain of type IV collagen $(7,40,41)$. The NC1 hexamer-containing fraction from each of these XAS kidneys was then analyzed by SDS-PAGE, immunoblotting, and direct ELISA.

SDS-PAGE analysis revealed NC1 dimers and monomers 54 and $27 \mathrm{kD}$ in size, respectively (data not shown). ELISA and immunoblotting experiments with $\mathrm{NC1}$ hexamer from XAS and normal kidneys using $\alpha$-chain-specific antibodies re- vealed the presence of all six isoforms of type IV collagen in normal kidneys (data not shown). Similar analysis of NC1 hexamers isolated from XAS kidney exhibited only residual amounts of $\alpha 3(\mathrm{IV}), \alpha 4(\mathrm{IV}), \alpha 5(\mathrm{IV})$, and $\alpha 6(\mathrm{IV})$ chains of type IV collagen in one kidney and complete absence of these chains in the four other XAS kidneys (data not shown). Similar to Immunohistochemical localization in XAS kidneys, the $\alpha 1$ (IV) and $\alpha 2(\mathrm{IV})$ chains were present in large amounts in type IV collagen extracted from XAS kidneys.

To further analyze the content of basement membrane collagens from XAS and normal kidneys, high resolution twodimensional gel electrophoresis and immunoblotting was performed using posttransplant Alport alloantibodies reactive to $\alpha 5(\mathrm{IV})$ and $\alpha 3(\mathrm{IV}) \mathrm{NC1}$ domains, and a Goodpasture autoantibody reactive to $\alpha 3$ (IV) $\mathrm{NC1}$ domain $(27,32)$. These antibody specificities are of particular importance as $\sim 10 \%$ of Alport patients that undergo renal transplantation develop posttransplant anti-GBM disease, with both circulating and kidney bound anti- $\alpha 3(\mathrm{IV}) \mathrm{NC1}$ and/or $-\alpha 5$ (IV) $\mathrm{NC1}$ antibodies $(27,42,43)$. The two-dimensional electrophoresis studies shown in Fig. 2 demonstrates that normal adult kidney express both antigens, while XAS kidney expresses neither $\alpha 3$ (IV) nor $\alpha 5$ (IV) chains. Typically, a much larger amount $(20 \mu \mathrm{g})$ of $\mathrm{NC} 1$ hexamer is used for the two-dimensional analysis as compared with $1 \mu \mathrm{g}$ in one-dimensional analyses. In one-dimensional analyses, we did not find any binding of Goodpasture or Alport alloantibodies to the Alport $\mathrm{NC1}$ hexamer; therefore, the shadow bands seen on the two-dimensional analysis (Fig. 2, $C$ and $D$ ) are probably due to very low level antibody crossreactivity.

To quantify the different isoforms of type IV collagen in normal and XAS kidneys, noncollagenous domains from these organs were biochemically analyzed by quantitative C-18 re-

Table II. Immunofluorescent Antibody Localization in Human Kidney

\begin{tabular}{|c|c|c|c|c|c|c|c|c|c|c|}
\hline \multirow[b]{2}{*}{ Antibody } & \multicolumn{5}{|c|}{ Control kidney } & \multicolumn{5}{|c|}{ Alport kidney } \\
\hline & GBM & $\mathrm{TBM}$ & $\mathrm{BCBM}$ & MM & VBM & GBM & TBM & $\mathrm{BCBM}$ & MM & VBM \\
\hline$\alpha 1$ & $-/+$ & ++ & ++ & ++ & + & ++ & ++ & ++ & ++ & + \\
\hline$\alpha 2$ & $-/+$ & ++ & ++ & ++ & + & ++ & ++ & + & + & + \\
\hline$\alpha 3$ & ++ & + & $-/+$ & - & ++ & - & - & - & - & $-/+$ \\
\hline$\alpha 4$ & ++ & + & - & - & - & - & - & - & - & - \\
\hline$\alpha 5$ & ++ & $-/+$ & - & - & - & - & $-/+$ & - & - & - \\
\hline$\alpha 6$ & - & - & ++ & - & $-/+$ & - & - & - & - & - \\
\hline ALP-1 & ++ & + & - & - & $-/+$ & - & - & - & - & - \\
\hline ALP-2 & ++ & ++ & - & - & - & - & - & - & - & - \\
\hline GP-1 & ++ & + & - & - & + & - & $-/+$ & - & - & - \\
\hline GP-2 & ++ & + & $-/+$ & - & $-/+$ & - & - & - & - & - \\
\hline Laminin & ++ & ++ & + & + & $-/+$ & ++ & ++ & ++ & + & + \\
\hline $\mathrm{rC}$ & - & - & - & $-/+$ & - & - & - & - & - & - \\
\hline $\mathrm{hC}$ & - & - & - & $-/+$ & - & - & - & - & - & - \\
\hline
\end{tabular}

Alp, Alport alloantibodies; GP, Goodpasture autoantibodies; rC, rabbit control sera; hC, human control sera; GBM, glomerular basement membrane; TBM, tubular basement membrane, BCBM, bowman's capsule basement membrane, MM, mesangial matrix. VBM, vascular basement membrane. + , positive; ++ , strongly positive; - , negative, and $-/+$, weakly positive. 

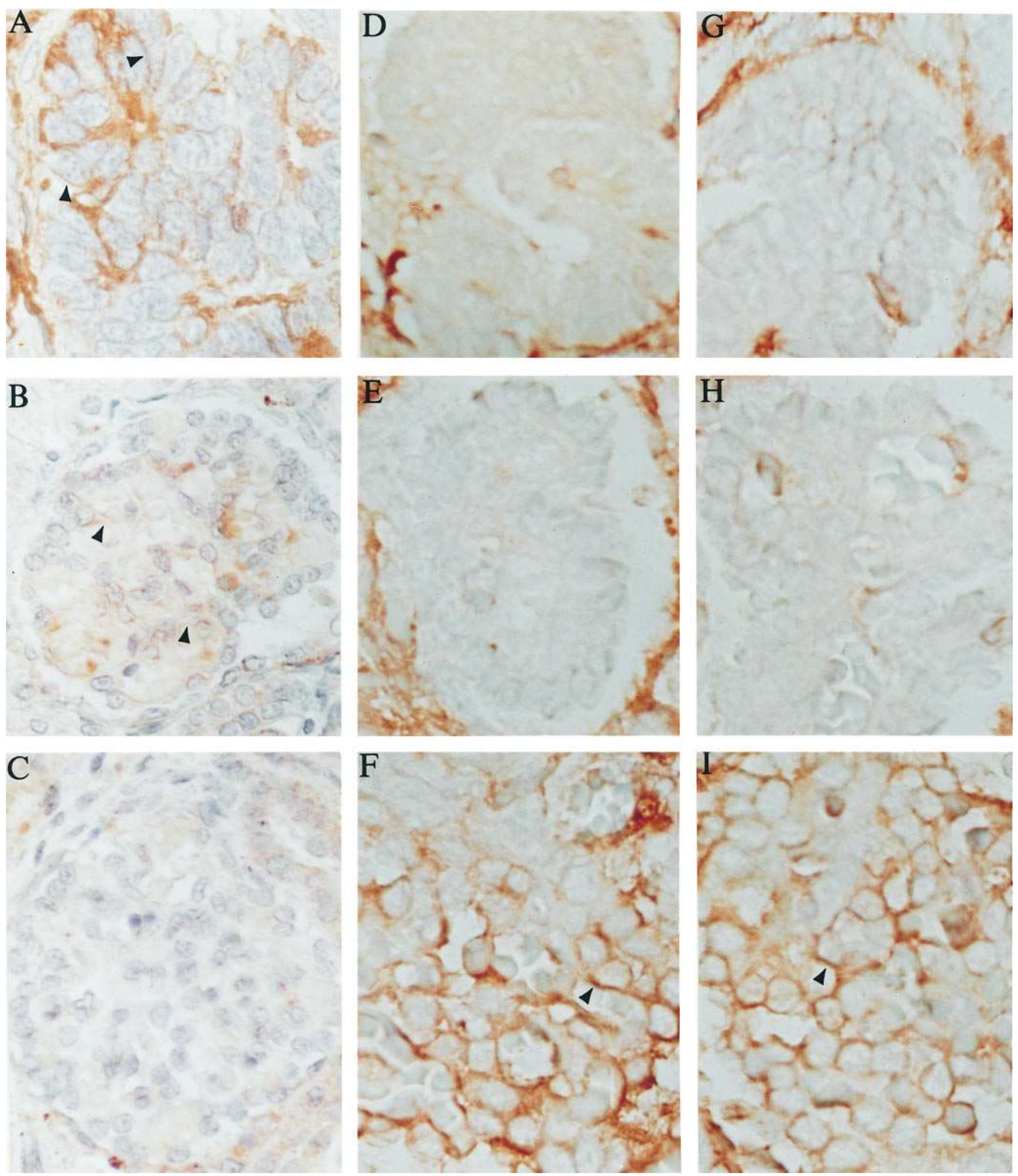

Figure 1. Expression of type IV collagen NC1 domain isoforms in developing human kidney. $A-C$ are probed with $\alpha 1$ (IV) antibody. $D-F$ are probed with Alport alloantibodies (27). $G-I$ are probed with $\alpha 5$ antibody. $A, D$, and $G$ represent the early cleft-shaped S-body. $B, E$, and $H$ represent early nephron and $C, F$, and $I$ represent mature nephron. All the sections were from a 75-d fetal human kidney and they were all developed using immunoperoxidase technique. The antibody dilutions were $\alpha 1,1: 700$; Alport alloantibodies, 1:500; $\alpha 5,1: 200$. $A-E$, $G$, and $H$, 250× and $F$ and $I, 400 \times$. 


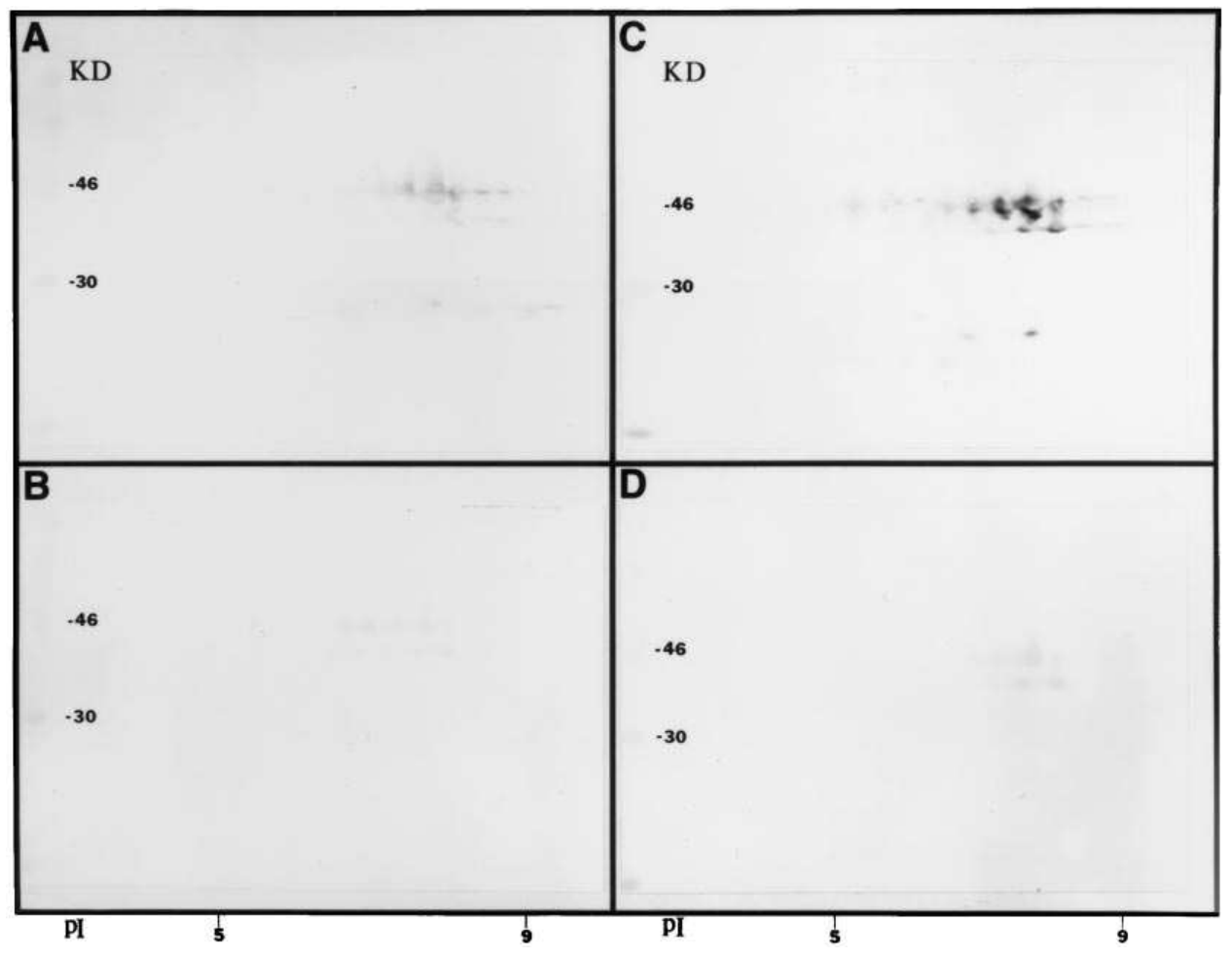

Figure 2. Two dimensional immunoblotting experiments with Alport alloantibodies and Goodpasture autoantibodies. Two dimensional immunoblotting was performed as described in Methods. Most of the spots that react to Goodpasture antibodies and Alport alloantibodies correspond to the highly cationic $\alpha 3$ spots identified by Derry and coworkers $(65,66)$. Some spots that react with Alport alloantibodies may be $\alpha 5$, as described previously by Hudson et al. (27) and Derry and co-workers $(65,66)$. $A$ and $C$ are control hexamer, and $B$ and $D$ are Alport hexamer. $A$ and $B$ are stained with Goodpasture autoantibodies, and $C$ and $D$ are stained with Alport alloantibodies. The antibody dilutions used were Alport alloantibodies, 1:1,000 and Goodpasture autoantibodies, 1:50. verse phase HPLC $(40,44)$. Four major peak areas were observed with normal NC1 hexamer (Fig. 3). In contrast, only two major peak areas could be observed with the XAS NC1 hexamer (Fig. 3). Quantitating the respective peak areas from both HPLC analyses revealed that peak areas III and IV in the XAS hexamer comprised only $4 \%$ of the total XAS NC1 hexamer, whereas in the normal kidneys this area constituted $26 \%$ (Fig. 3).

To identify the proteins in these peaks, larger amounts of these NC1-containing proteins were resolved by C-18 HPLC and analyzed by direct ELISA and immunoblotting. All four peaks were collected from normal NC1 hexamer and XAS NC1 hexamer. These fractions were analyzed by $\alpha$-chain-spe- cific antibodies to the six different chains of type IV collagen ( $\alpha 1-\alpha 6)$. By ELISA, in the C-18 fractions from normal kidneys, pool I contained $\alpha 1$ (IV) and $\alpha 2$ (IV); pool II contained $\alpha 1(\mathrm{IV}), \alpha 2(\mathrm{IV})$, and $\alpha 5(\mathrm{IV})$; pool III contained $\alpha 4(\mathrm{IV})$ and $\alpha 6(\mathrm{IV})$; and pool IV contained $\alpha 3$ (IV) NC1 domain (Fig. 3). ELISA analysis of XAS C-18 fractions revealed that $\alpha 5$ (IV) was missing from pool II, $\alpha 4$ (IV) and $\alpha 6$ (IV) were not present in pool III, and $\alpha 3$ (IV) was absent in pool IV (Fig. 3). SDSPAGE analysis of these fractions revealed that pool I contained NC1 monomers, pool II contained NC1 dimers, and pools III and IV contained a mixture of monomers and dimers (data not shown) $(44,45)$.

The XAS NC1 hexamer analysis revealed monomers and

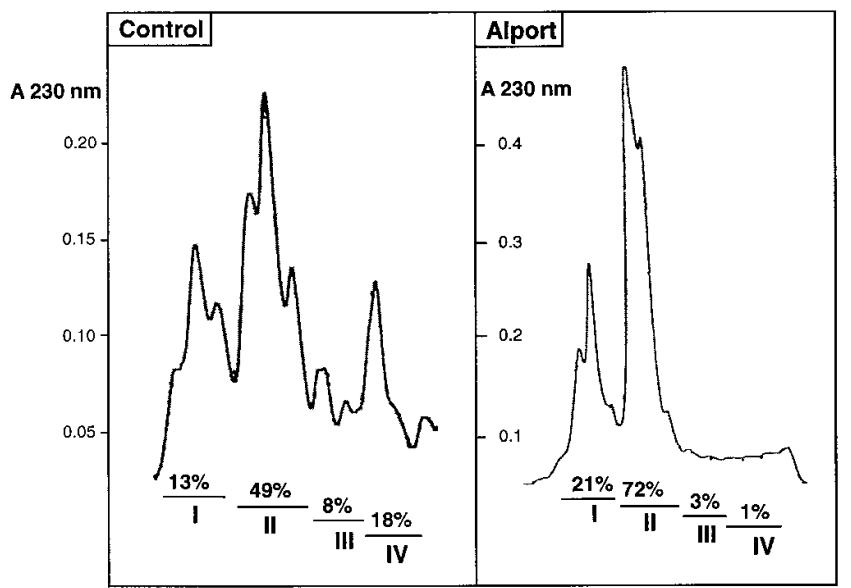

Figure 3. C-18 reverse-phase HPLC quantification of type IV collagen NC1 domains in Alport and control human kidneys. Reverse-phase HPLC was performed as described in Methods. For qualitative analysis, $250 \mu \mathrm{g}$ of DEAE-52 unbound concentrated material was loaded on a C-18 column that separates proteins based on their hydrophobicity. For quantitative assessment, $10 \mathrm{mg}$ of the same material was loaded and different peaks collected separately. The collected peaks were lyophilized and solubilized in $50 \mathrm{mM}$ Tris-Cl, $\mathrm{pH} 7.5$, solution and used in ELISA and immunoblot analysis. Direct ELISA was performed as previously described (31). The dashed lines in the ELISA analysis indicate no immunoreactivity. 


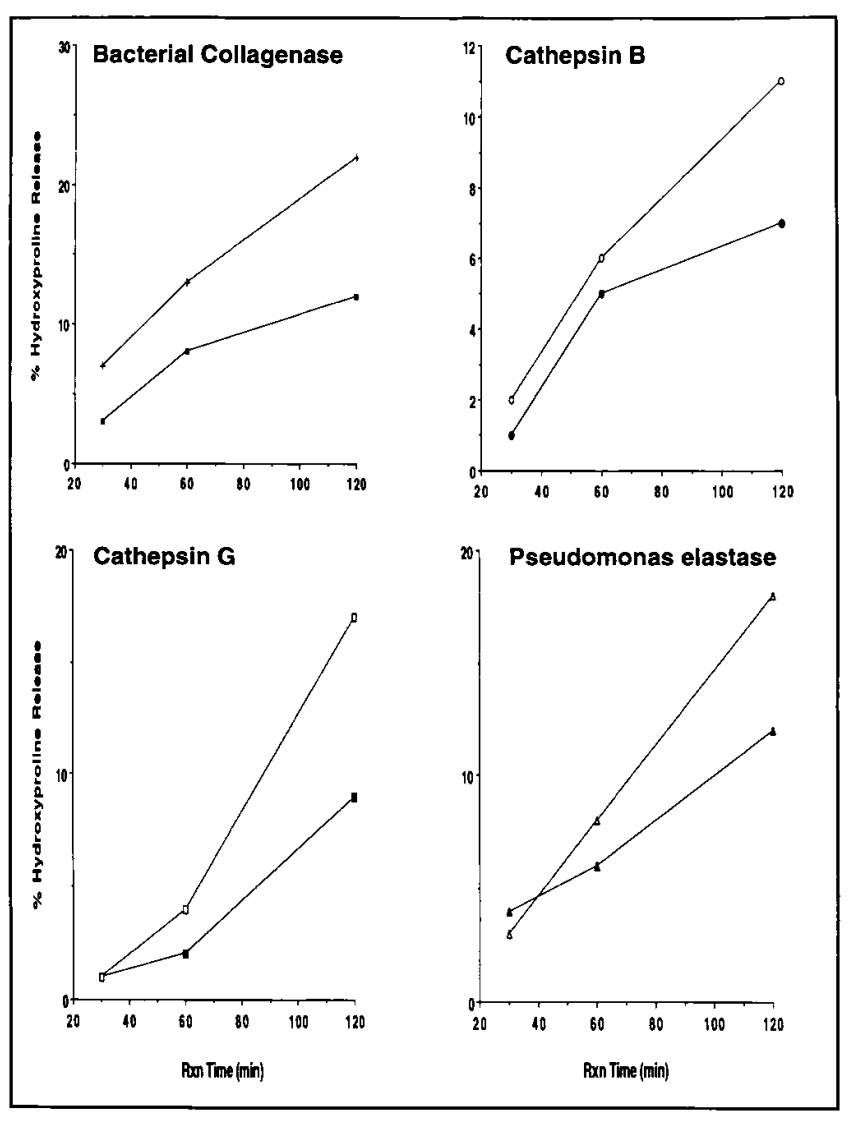

Figure 4. Hydroxyproline release assay. $1 \mathrm{mg}$ dry detergentextracted renal basement membranes from control and Alport kidney was digested with different enzymes for $2 \mathrm{~h}$. Aliquots were collected at 30 and $60 \mathrm{~min}$ after initiating the digestion. Hydroxyproline release assay was performed as described previously $(19,36)$. The percent hydroxyproline release was quantitated as described by Shah et al. (46). For bacterial collagenase digestion, the X denotes XAS $\mathrm{RBM}$ digestion and the dark rectangles denote control digestion. For cathepsin B digestion, open circles denote XAS RBM digestion and dark circles denote control RBM digestion. For cathepsin $\mathrm{G}$ digestion, open rectangles denote XAS RBM digestion and dark rectangles denote control RBM digestion. For $P$. elastase digestion, open triangles denote XAS RBM digestion and dark triangles denote control RBM digestion. Incubation of XAS or normal kidney basement membrane preparations in buffer did not result in an accumulation of collagen fragments in the supernatant (data not shown). The experiment was performed separately three times and the values in the graph are an average of the three experiments.

dimers in pools I and II only. No protein was detected in fractions III and IV (data not shown). Immunoblotting analysis of the $\mathrm{C}-18$ fractions from normal and XAS hexamers revealed results similar to those observed by ELISA (data not shown). The $\alpha 3$ (IV) antibody bound predominantly to the pool IV protein fraction from normal kidney. The anti- $\alpha 4$ (IV) antibody binds to pool III, anti- $\alpha 5$ (IV) binds to pool II, and anti- $\alpha 6$ (IV) binds to pool III with some minor reactivity to pool I. No $\alpha 3(\mathrm{IV}), \alpha 4(\mathrm{IV}), \alpha 5(\mathrm{IV})$, or $\alpha 6(\mathrm{IV})$ antibody reactivity was observed with pool I-IV fractions from XAS hexamers. In both normal and XAS hexamer fractions, $\alpha 1$ (IV) and $\alpha 2$ (IV) chains were present by ELISA and immunoblotting (data not shown).

To determine the contribution of $\alpha 3$ (IV), $\alpha 4(\mathrm{IV})$, and

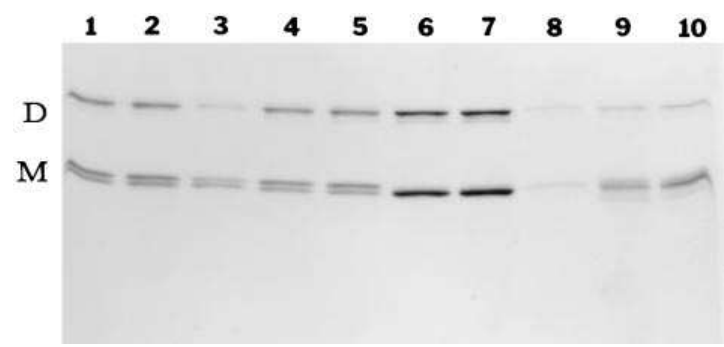

Figure 5. Type IV collagen NC1 hexamer digestion with endoproteinases. NC1 hexamer was prepared from concentrated DE-52 unbound material $(5 \mathrm{mg}$ ) resolved on a Bio Sil TSK-250 HPLC gel filtration column (7). $10 \mu \mathrm{g} \mathrm{NC1}$ hexamer was digested with various proteinases. The digestion was terminated at $15 \mathrm{~min}$. One fifth of the each sample analyzed by $10 \%$ SDS-PAGE and stained with Coomassie blue. Lane 1, normal hexamer (NH); lane 2, NH-trypsin; lane 3, NH-pepsin; lane 4, NH-cathepsin G; lane 5, NH-cathepsin B; lane 6, Alport Hexamer (AH); lane 7, AH-trypsin; lane 8, AH-pepsin; lane 9, AH-cathepsin G; lane 10, AH-cathepsin B. D, NC1 dimers $(\sim 54 \mathrm{kD}) ; \mathrm{M}, \mathrm{NC} 1$ monomers $(\sim 28 \mathrm{kD})$.

$\alpha 5$ (IV) chains of type IV collagen to the structural integrity of GBM and other kidney basement membranes, we performed timed digestions using different endopeptidases. These analyses used hydroxyproline release as an assessment of triple helical collagen degradation $(19,46)$. Detergent-extracted XAS kidney basement membrane was more readily degradable by bacterial collagenase, $P$. elastase, cathepsin $\mathrm{B}$, and cathepsin $\mathrm{G}$ (Fig. 4, $A-D$ ), compared with preparations of normal kidney basement membrane; XAS versus normal basement membranes after $2 \mathrm{~h}$ degradation: bacterial collagenase, $22 \pm 4 \%$ vs. $12 \pm 1 \%(P \leq 0.04) ; P$. elastase, $18 \pm 2 \%$ vs. $12 \pm 1.5 \%(P \leq$ $0.05)$; cathepsin $\mathrm{B}, 11 \pm 0.5 \%$ vs. $7 \pm 0.3 \%(P \leq 0.03)$; cathepsin G, $17 \pm 1.5 \%$ vs. $9 \pm 0.8 \%(P \leq 0.01)$. These results strongly suggest that the presence of $\alpha 3$ (IV), $\alpha 4(\mathrm{IV})$, and $\alpha 5$ (IV) collagens in kidney basement membranes retard in vitro proteolysis. Additionally, type IV collagen NC1 hexamer from XAS and normal kidneys were also subjected to proteolysis by several endopeptidases. After 15 min incubation, pepsin was the only enzyme that digested the normal GBM hexamer to a measurable degree (Fig. 5). The XAS hexamer was digested by pepsin, cathepsin B, and cathepsin $\mathrm{G}$. These results suggest that basement membrane collagens, even at the hexamer level, have greater resistance to proteolysis when comprised of $\alpha 3$ (IV), $\alpha 4$ (IV), and $\alpha 5$ (IV) chains.

Finally, we evaluated the ability of pooled conditioned medium from glomerular epithelial, endothelial, and mesangial cells to degrade detergent-extracted RBM from human kidneys. The results from three separate experiments indicate that RBM from XAS kidneys released more hydroxyproline with glomerular cell supernatants then RBM from normal kidneys $(P \leq 0.03)$; RBM from diabetic and IgA nephropathy kidneys were no different then normal ( $P=$ NS) (Fig. 6). By immunofluorescence, the normal, diabetic, and IgA nephropathy kidneys expressed $\alpha 3, \alpha 4$, and $\alpha 5$ chains of type IV collagen along their GBM (data not shown).

\section{Discussion}

Our findings suggest that the switch from fetal $\alpha 1$ (IV) and $\alpha 2(\mathrm{IV})$ collagen chains to $\alpha 3$ (IV), $\alpha 4(\mathrm{IV})$, and $\alpha 5$ (IV) chains 


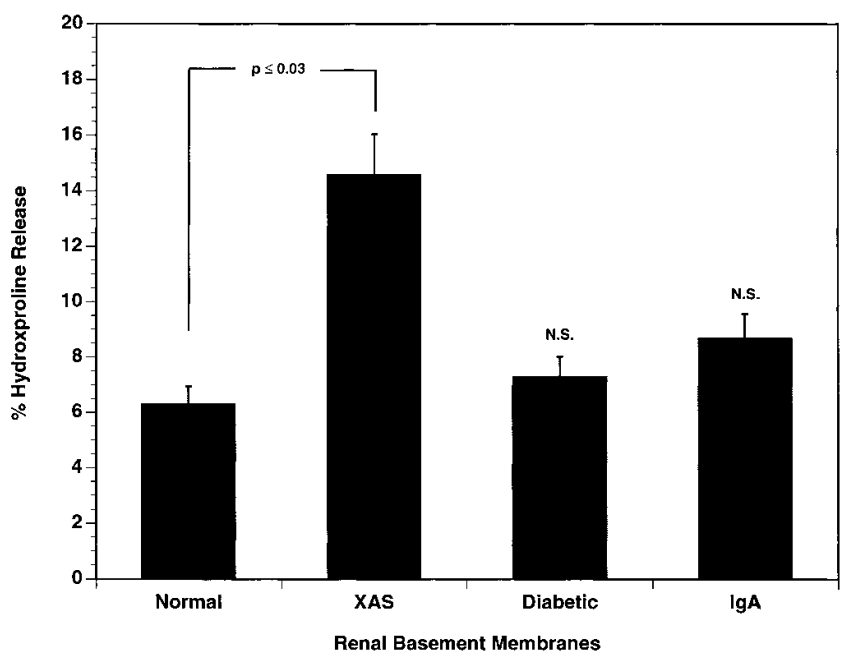

Figure 6. Degradation of renal basement membranes by the proteases generated by rat glomerular epithelial, endothelial, and mesangial cells. $1 \mathrm{mg}$ of dry detergent-extracted renal basement membranes from control, Alport, diabetic, and IgA nephropathy kidney was incubated in $15 \mathrm{ml}$ pooled conditioned medium from the three glomerular cell types for $12 \mathrm{~h}$. Hydroxyproline release assay was performed as described elsewhere $(19,36)$. The percent hydroxyproline release was quantitated as described by Shah et al. (46). The experiment was performed separately three times and the values in the graph are an average of the three experiments.

along human GBM is an early developmental event. In XAS this switch does not occur, or in some recent cases has shown variable penetrance (12). While the transcriptional basis for this developmental switch is not known or addressed by the current work, we did consider a central question of why such a switch might be favored in renal tissue.

The idea that GBM collagens might go through a developmental switch was first suggested in rodents (47). This observation seems to be species-independent, and the notion that specialized basement membranes in XAS are in developmental arrest, in retrospect, can be partially inferred from earlier work (48) and established by ours. Several recent antibody staining experiments, for example, have shown that the type IV collagen in normal GBM is represented almost exclusively by $\alpha 3$ (IV), $\alpha 4$ (IV), and $\alpha 5$ (IV) chains $(5,47,49)$. Immunofluorescent studies using Goodpasture antibodies directed to the $\alpha 3$ (IV) chain demonstrate its absence in kidney biopsies from Alport patients (50). Recent studies also demonstrate absent or aberrant expression of $\alpha 4$ (IV), $\alpha 5$ (IV), and $\alpha 6$ (IV) chains in XAS kidneys (13). Finally, collagenase digestion of Alport kidneys previously suggested the absence of certain polypeptides in the range of $28 \mathrm{kD}$, compared with similarly digested normal kidney (51). These bands are now inferred to be the $\alpha 3$ (IV) chain and possibly the $\alpha 4$ (IV) collagen chain. Our studies of immunofluorescence on kidneys from four different patients with XAS are consistent with these findings, and our reverse-phase analysis and Western blotting of the collagen chains did not detect $\alpha 3$ (IV), $\alpha 4$ (IV), $\alpha 5$ (IV), or $\alpha 6$ (IV) chains. We surmise that the increased amount of $\alpha 1$ (IV) and $\alpha 2$ (IV) collagens in XAS kidneys was proportionally greater than normal, but did not exceed the total collagen mass expected from normal kidney basement membranes.
We hypothesize that the developmental switch and normal replacement of fetal collagen chains must be fundamentally important to the long term stability of GBM in its role as a filter. One explanation for this switch is that kidney basement membranes containing $\alpha 3$ (IV), $\alpha 4$ (IV), and $\alpha 5$ (IV) collagen chains and $\mathrm{NC} 1$ hexamers are more resistant to endopeptidase digestion than $\alpha 1$ (IV) and $\alpha 2$ (IV) chains found in XAS kidneys. $\alpha 3(\mathrm{IV})$ and $\alpha 4(\mathrm{IV})$ collagen chains are more cysteine rich in their $7 \mathrm{~S}$ and triple helical regions than $\alpha 1$ (IV) or $\alpha 2$ (IV) chains, possibly enabling them to form more disulfide bonds leading to more covalent cross-links $(5,52)$. In this regard, type IV collagen protomers of $\alpha 1$ (IV) and $\alpha 2$ (IV) bovine GBM are more easily extracted by $P$. elastase than $\alpha 3$ (IV)-, $\alpha 4(\mathrm{IV})-$, and $\alpha 5$ (IV)-containing protomers (53), and protomers of $\alpha 1$ (IV) and $\alpha 2(\mathrm{IV})$ dimerize through $\mathrm{NC1}-\mathrm{NC} 1$ interactions, while protomers containing $\alpha 3$ (IV), $\alpha 4$ (IV), and $\alpha 5$ (IV) exist as a supramolecular complex with $\mathrm{NC1}-\mathrm{NC} 1$ interactions and interprotomer disulfide bonds between triple helices (53).

The bidirectional influence of extracellular matrix on cell differentiation has been suggested for some time (54-56). In nearly all forms of Alport syndrome, defective composition of GBM has been implicated in the pathogenesis of hematuria and proteinuria leading to eventual renal failure $(5,8,10)$. We believe, as a hypothesis, that the relative persistence of fetal isoforms of type IV collagen in GBM, or the incomplete conversion to more mature isoforms, affects glomerular cell integrity, the onset of clinical disease, and/or the rate of progression to end-stage kidney.

We further surmise that the greater vulnerability to deterioration of GBM in XAS is a local event. Several endopeptidases are secreted by cells surrounding the GBM, and the circulation likely carries such moieties in varying states of activation. Prominent among these endopeptidases are basement membrane-degrading enzymes such as collagenase, elastase, cathepsins, and metalloproteinases (19-22, 24, 46, 57-63). We tested in this report three of these classes of enzymes, and when biologically active metalloproteinases become more readily available, they will be important to study in this condition. In this regard, several recent experiments performed by various groups indicate that glomerular epithelium and mesangial cells produce metalloproteinases, neutral proteases, and cathepsins (20-22, 24, 37, 38, 63, 64). We also tested for RBM degradation by proteases in conditioned media from mesangial, glomerular epithelial, and glomerular endothelial cells. It appears, as was observed for the defined proteases mentioned above, that secreted glomerular cell proteases also degrade XAS RBM more readily than the RBM from normal, diabetic, or IgA nephropathy kidneys.

We do not believe that the age or condition of the kidneys used in the present study had any likely bearing on our results, as we used only intact type IV collagen for our analysis and, additionally, kidneys from patients with diabetes and $\operatorname{Ig} \mathrm{A}$ nephropathy showed comparable rates of degradation as the normal human kidney. Undoubtedly there may be less type IV collagen in older XAS kidneys, but those that remain should be representative of the total. Additionally, incubation of XAS or normal kidney basement membrane preparations in buffer did not result in an accumulation of collagen fragments in the supernatant, suggesting that XAS basement membranes are not more prone to spontaneous autoproteolysis.

A consideration of the differential sensitivity of GBM in XAS to endopeptidases might eventually warrant a compara- 
tive analysis of collagen fragments in the urine of patients with XAS and perhaps at some point a therapeutic trial with the new generation of protease inhibitors now emerging for clinical use.

\section{Acknowledgments}

The technical assistance of Michelle C. Werner and Mark B. Ericksen with immunofluorescence experiments and cell culture, respectively, is appreciated.

This work was supported in part by National Institutes of Health grants DK-30280, DK-45191, DK-07006, and DK-18381, and the DCI RED Fund.

\section{References}

1. Maddox, D.A., and B.M. Brenner. 1996. Glomerular Ultrafiltration. In The Kidney. Vol. 1. B.M. Brenner, editor. W.B. Saunders Co., Philadelphia, PA. 286-333.

2. Elger, M., B. Kaissling, M. Le Hir, and W. Kriz. 1997. Microanatomy of the kidney: vessels, interstitium, and glomerulus. In Immunologic Renal Diseases. E.G. Neilson and W.G. Couser, editors. Lippincott-Raven, Philadelphia. $15-42$.

3. Abrahamson, D.R. 1987. Stucture and development of the glomerular capillary wall and basement membrane. Am. J. Physiol. 22:F783.

4. Timpl, R. 1989. Strucure and biological activities of basement membrane proteins. Eur. J. Biochem. 180:487-502.

5. Hudson, B.G., S.T. Reeders, and K. Tryggvason. 1993. Type IV collagen: structure, gene organization, and role in human diseases. Molecular basis of Goodpasture and Alport syndromes and diffuse leiomyomatosis. J. Biol. Chem. 268:26033-26036.

6. Enders, G.C., T.Z. Kahsai, G. Lian, K. Funabiki, P.D. Killen, and B.G. Hudson. 1995. Developmental changes in seminiferous tubule extracellular matrix components of the mouse testis: alpha 3(IV) collagen chain expressed at the initiation of spermatogenesis. Biol. Reprod. 53:1489-1499.

7. Gunwar, S., F. Ballester, R. Kalluri, J. Timoneda, A.M. Chonko, S.J. Edwards, M.E. Noelken, and B.G. Hudson. 1991. Glomerular basement membrane: identification of dimeric subunits of the noncollagenous domain (hexamer) of collagen IV and the Goodpasture antigen. J. Biol. Chem. 266:15318-15324.

8. Tryggvason, K., J. Zhou, S.L. Hostikka, and T.B. Shows. 1993. Molecular genetics of Alport syndrome. Kidney Int. 43:38-44.

9. Kashtan, C.E., and A.F. Michael. 1993. Alport syndrome: from bedside to genome to bedside. Am. J. Kidney Dis. 22:627-640.

10. Kashtan, C.E., R.K. Sibley, A.E. Michael, and R.L. Vernier. 1994. Hereditary Nephritis: Alport Syndrome and Thin Glomerular Basement Membrane Disease. J. B. Lippincott Co., Philadelphia, PA. 1239-1266.

11. Grunfeld, J.-P. 1985. The clinical spectrum of hereditary nephritis. Kidney Int. 27:83.

12. Thorner, P.S., K. Zheng, R. Kalluri, R. Jacobs, and B.G. Hudson. 1996. Coordinate gene expression of $\alpha 3, \alpha 4$ and $\alpha 5$ chains of collagen type IV. J. Biol. Chem. 271:13821-13828.

13. Nakanishi, K., N. Yoshikawa, K. Iijima, K. Kitagawa, H. Nakamura, H. Ito, K. Yoshioka, M. Kagawa, and Y. Sado. 1994. Immunohistochemical study of alpha 1-5 chains of type IV collagen in hereditary nephritis. Kidney Int. 46: 1413-1421.

14. Wolf, G., F.N. Ziyadeh, G. Zahner, and R.A. Stahl. 1996. Angiotensin II is mitogenic for cultured rat glomerular endothelial cells. Hypertension (Dallas). 27:897-905.

15. Singh, A.K. 1993. Presence of lysosomal enzymes in the normal glomerular basement membrane matrix. Histochem. J. 25:562-568.

16. Tryggvason, K., J. Risteli, and K.I. Kivirikko. 1978. Glomerular basement membrane collagen and activities of the intracellular enzymes of collagen biosynthesis in congenital nephrotic syndrome of the Finnish type. Clin. Chim. Acta. 82:233-240.

17. Walker, F. 1973. The origin, turnover and removal of glomerular basement-membrane. J. Pathol. 110:233-244.

18. Baricos, W.H., S.L. Cortez, Q.C. Le, Y.W. Zhou, R.M. Dicarlo, S.E. O'Connor, and S.V. Shah. 1990. Glomerular basement membrane degradation by endogenous cysteine proteinases in isolated rat glomeruli. Kidney Int. 38: 395-401.

19. Baricos, W.H., Y. Zhou, R.W. Mason, and A.J. Barrett. 1988. Human kidney cathepsins B and L. Characterization and potential role in degradation of glomerular basement membrane. Biochem. J. 252:301-304.

20. Davies, M., A.J. Barrett, J. Travis, E. Sanders, and G.A. Coles. 1978. The degradation of human glomerular basement membrane with purified lysosomal proteinases: evidence for the pathogenic role of the polymorphonuclear leucocyte in glomerulonephritis. Clin. Sci. Mol. Med. 54:233-240.
21. Davies, M., K.T. Hughes, and G.J. Thomas. 1980. Evidence that kidney lysosomal proteinases degrade the collagen of glomerular basement membrane. Renal Physiol. 3:116-119.

22. Davies, M., G.A. Coles, G.J. Thomas, J. Martin, and D.H. Lovett. 1990. Proteinases and the glomerulus: their role in glomerular diseases. Klin. Wochenschr. 68:1145-1149.

23. Donovan, K.L., M. Davies, G.A. Coles, and J.D. Williams. 1994. Relative roles of elastase and reactive oxygen species in the degradation of human glomerular basement membrane by intact human neutrophils. Kidney Int. 45: 1555-1561.

24. Knowlden, J., J. Martin, M. Davies, and J.D. Williams. 1995. Metalloproteinase generation by human glomerular epithelial cells. Kidney Int. 47: 1682-1689.

25. Peissel, B., L. Geng, R. Kalluri, C. Kashtan, H.G. Rennke, G.R. Gallo, K. Yoshioka, M.J. Sun, B.G. Hudson, E.G. Neilson, et al. 1995. Comparative distribution of the alpha (IV), alpha 5(IV), and alpha 6(IV) collagen chains in normal human adult and fetal tissues and in kidneys from X-linked Alport syndrome patients. J. Clin. Invest. 96:1948-1957.

26. Reddy, G.K., S. Gunwar, R. Kalluri, B.G. Hudson, and M.E. Noelken. 1993. Structure and composition of type IV collagen of bovine aorta. Biochim. Biophys. Acta. 1157:241-251.

27. Hudson, B.G., R. Kalluri, S. Gunwar, M. Weber, F. Ballester, J.K. Hudson, M.E. Noelken, M. Sarras, W.R. Richardson, and J. Saus. 1992. The pathogenesis of Alport syndrome involves type IV collagen molecules containing the alpha 3(IV) chain: evidence from anti-GBM nephritis after renal transplantation. Kidney Int. 42:179-187.

28. West, T.W., J.W. Fox, M. Jodlowski, J.W. Freytag, and B.G. Hudson. 1980. Bovine glomerular basement membrane. J. Cell Biol. 255:10451-10459.

29. Wieslander, J., M. Kataja, and B.G. Hudson. 1987. Characterization of the human Goodpasture antigen. Clin. Exp. Immunol. 69:332-340.

30. Timoneda, J., S. Gunwar, G. Monfort, J. Saus, M.E. Noelken, and B.G. Hudson. 1990. Unusual dissociative behavior of the noncollagenous domain (hexamer) of basement membrane collagen during electrophoresis and chromatofocusing. Connect. Tissue Res. 24:169-186.

31. Kalluri, R., M. Sun, B. Hudson, and E.G. Neilson. 1996. The Goodpasture autoantigen: structural delineation of two immunologically privileged epitopes on $\alpha 3$ (IV) chain of type IV collagen. J. Biol. Chem. 271:9062-9068.

32. Kalluri, R., C.B. Wilson, M. Weber, S. Gunwar, A.M. Chonko, E.G. Neilson, and B.G. Hudson. 1995. Identification of the $\alpha 3(\mathrm{IV})$ chain of type IV collagen as the common autoantigen in anti-basement membrane disease and Goodpasture syndrome. J. Am. Soc. Nephrol. 6:1178-1185.

33. Haberstroh, U., J. Zahner, M. Disser, F. Thaiss, G. Wolf, and R.A. Stahl. 1993. TGF-beta stimulates rat mesangial cell proliferation in culture: role of PDGF beta-receptor expression. Am. J. Physiol. 264:F199-205.

34. Coers, W., S. Huitema, R.J. Smeenk, D.J. Salant, J. Grond, and J.J. Weening. 1992. Quantification of glomerular epithelial cell adhesion by using anti-DNA antibodies in ELISA. Hybridoma. 11:529-537.

35. O'Meara, Y.M., Y. Natori, A.W. Minto, D.J. Goldstein, E.C. Manning, and D.J. Salant. 1992. Nephrotoxic antiserum identifies a beta 1-integrin on rat glomerular epithelial cells. Am. J. Physiol. 262:F1083-1091.

36. Huszar, G., J. Maiocco, and F. Naftolin. 1980. Monitoring of collagen and collagen fragments in chromatography of protein mixtures. Anal. Biochem. 105:424-429.

37. Martin, J., M. Davies, G. Thomas, and D.H. Lovett. 1989. Human mesangial cells secrete a GBM-degrading neutral proteinase and a specific inhibitor. Kidney Int. 36:790-801.

38. Lovett, D.H., R.B. Sterzel, M. Kashgarian, and J.L. Ryan. 1983. Neutral proteinase activity produced in vitro by cells of the glomerular mesangium. Kidney Int. 23:342-349.

39. Butkowski, R.J., J. Wieslander, M. Kleppel, A.F. Michael, and A.J. Fish. 1989. Basement membrane collagen in the kidney: regional localization of novel chains related to collagen IV. Kidney Int. 35:1195-1202.

40. Butkowski, R.J., J. Wieslander, B.J. Wisdom, J.F. Barr, M.E. Noelken, and B.G. Hudson. 1985. Properties of the globular domain of type IV collagen and its relationship to the Goodpasture antigen. J. Biol. Chem. 260:3739-3747.

41. Wisdom, B.J.J., S. Gunwar, M.D. Hudson, M.E. Noelken, and B.G. Hudson. 1992. Type IV collagen of Engelbreth-Holm-Swarm tumor matrix: identification of constituent chains. Connect. Tissue Res. 27:225-234.

42. Kalluri, R., L.P. van den Heuvel, H.J. Smeets, C.H. Schroder, H.H. Lemmink, A. Boutaud, E.G. Neilson, and B.G. Hudson. 1995. A COL4A3 gene mutation and post-transplant anti-alpha 3(IV) collagen alloantibodies in Alport syndrome. Kidney Int. 47:1199-1204.

43. Kalluri, R., M. Weber, K.O. Netzer, M.E. Sun, E.G. Neilson, and B.G. Hudson. 1993. COL4A5 gene deletion and production of postransplant anti$\alpha 3$ (IV) alloantibodies in Alport syndrome. Kidney Int. 45:721-726.

44. Gunwar, S., P.A. Bejarano, R. Kalluri, J.P. Langeveld, B.J.J. Wisdom, M.E. Noelken, and B.G. Hudson. 1991. Alveolar basement membrane: molecular properties of the noncollagenous domain (hexamer) of collagen IV and its reactivity with Goodpasture autoantibodies. Am. J. Respir. Cell Mol. Biol. 5: 107-112.

45. Langeveld, J.P., J. Wieslander, J. Timoneda, P. McKinney, R.J. Butkowski, B.J. Wisdom, and B.G. Hudson. 1988. Structural heterogeneity of the 
noncollagenous domain of basement membrane collagen. J. Biol. Chem. 263: 10481-10488.

46. Shah, S.V., W.H. Baricos, and A. Basci. 1987. Degradation of human glomerular basement membrane by stimulated neutrophils. Activation of a metalloproteinase(s) by reactive oxygen metabolites. J. Clin. Invest. 79:25-31.

47. Miner, J.H., and J.R. Sanes. 1994. Collagen IV alpha 3, alpha 4, and alpha 5 chains in rodent basal laminae: sequence, distribution, association with laminins, and developmental switches. J. Cell Biol. 127:879-891.

48. Kleppel, M.M., and A.F. Michael. 1990. Expression of novel basement membrane components in the developing human kidney and eye. Am. J. Anat. 187:165-174.

49. Sugimoto, M., T. Oohashi, and Y. Ninomiya. 1994. The genes COL4A5 and COL4A6, coding for basement membrane collagen chains alpha 5(IV) and alpha 6(IV), are located head-to-head in close proximity on human chromosome Xq22 and COL4A6 is transcribed from two alternative promoters. Proc. Natl. Acad. Sci. (USA). 91:11679-11683.

50. McCoy, R.C., H.K. Johnson, W.J. Stone, and C.B. Wilson. 1982. Absence of nephritogenic GBM antigen(s) in some patients with hereditary nephritis. Kidney Int. 21:642-652.

51. Kashtan, C., A.J. Fish, M.M. Kleppel, K. Yoshioka, and A.F. Michael. 1986. Nephritogenic antigen determinants in epidermal and renal basement membrane of kindreds with Alport-type familial nephritis. J. Clin. Invest. 78: $1035-1044$.

52. Gunwar, S., M.E. Noelken, and B.G. Hudson. 1991. Properties of the collagenous domain of the alpha 3(IV) chain, the Goodpasture antigen, of lens basement membrane collagen. Selective cleavage of alpha (IV) chains with retention of their triple helical structure and noncollagenous domain. J. Biol. Chem. 266:14088-14094.

53. Gunwar, S., F. Ballester, M.E. Noelken, and B.G. Hudson. 1992. Characterization of a supramolecular complex composed of the $\alpha 3, \alpha 4$ and $\alpha 5$ chains of type IV collagen. Implications for the pathogenesis of Alport syndrome. $J$. Am. Soc. Nephrol. 3:296.

54. Guan, J.L., and H.C. Chen. 1996. Signal transduction in cell-matrix interactions. Int. Rev. Cytol. 168:81-121.

55. Lin, C.Q., and M.J. Bissell. 1993. Multi-faceted regulation of cell differentiation by extracellular matrix. FASEB J. 7:737-743.

56. Kleinman, H.K., M.L. McGarvey, J.R. Hassell, V.L. Star, F.B. Cannon,
G.W. Laurie, and G.R. Martin. 1986. Basement membrane complexes with biological activity. Biochemistry. 25:312-318.

57. Brodt, P., R. Reich, L.A. Moroz, and A.F. Chambers. 1992. Differences in the repertoires of basement membrane degrading enzymes in two carcinoma sublines with distinct patterns of site-selective metastasis. Biochim. Biophys. Acta. 1139:77-83.

58. Walker, P.D., G.P. Kaushal, and S.V. Shah. 1994. Presence of a distinct extracellular matrix-degrading metalloproteinase activity in renal tubules. $J$. Am. Soc. Nephrol. 5:55-61.

59. Kaushal, G.P., P.D. Walker, and S.V. Shah. 1994. An old enzyme with a new function: purification and characterization of a distinct matrix-degrading metalloproteinase in rat kidney cortex and its identification as meprin. J. Cell Biol. 126:1319-1327.

60. Davies, M., G.A. Coles, and K.T. Hughes. 1980. Glomerular basement membrane injury by neutrophil and monocyte neutral proteinases. Renal Physiol. 3:106-111.

61. Davies, M., G.J. Thomas, J. Martin, and D.H. Lovett. 1988. The purification and characterization of a glomerular-basement-membrane-degrading neutral proteinase from rat mesangial cells. Biochem. J. 251:419-425.

62. Hughes, K.T., G.A. Coles, T.R. Harry, and M. Davies. 1981. Some properties of human blood monocyte cell lysate neutral proteinase(s). Biochim. Biophys. Acta. 662:111-118

63. Lovett, D.H., R.J. Johnson, H.P. Marti, J. Martin, M. Davies, and W.G. Couser. 1992. Structural characterization of the mesangial cell type IV collagenase and enhanced expression in a model of immune complex-mediated glomerulonephritis. Am. J. Pathol. 141:85-98.

64. Vissers, M.C., and C.C. Winterbourn. 1988. Gelatinase contributes to the degradation of glomerular basement membrane collagen by human neutrophils. Collagen Relat. Res. 8:113-122.

65. Derry, C.J., M. Pickering, C. Baker, and C.D. Pusey. 1994. Identification of the Goodpasture antigen, alpha 3(IV) NC1, and four other NC1 domains of type IV collagen, by amino-terminal sequence analysis of human glomerular basement membrane separated by two-dimensional electrophoresis. Exp. Nephrol. 2:249-256.

66. Derry, C.J., and C.D. Pusey. 1994. Tissue-specific distribution of the Goodpasture antigen demonstrated by 2-D electrophoresis and Western blotting. Nephrol. Dial. Transplant. 9:355-361. 